九州大学学術情報リポジトリ

Kyushu University Institutional Repository

\title{
STATIC CHARACTERISTICS OF TRIANGULAR LOOP WITH NONIDENTICAL JOSEPHSON JUNCTIONS
}

Aomine, Takafumi

Research Institute of Fundamental Information Science, Kyushu University

Hidaka, Mutsuo

Department of Information Systems, Interdisciplinary Graduate School of Engineering Sciences, Kyushu University

Matsuo, Kazuhiro

International Institute for Advanced Study of Social Information Science, Fujitsu Ltd.,

Kitagawa, Toshio

International Institute for Advanced Study of Social Information Science, Fujitsu Ltd.,

https://doi.org/10.5109/13359

出版情報: Bulletin of informatics and cybernetics. 21 (1/2), pp.85-92, 1984-03. Research Association of Statistical Sciences

バージョン :

権利関係 : 


\title{
STATIC CHARACTERISTICS OF TRIANGULAR LOOP WITH NONIDENTICAL JOSEPHSON JUNCTIONS
}

\author{
By
Takafumi Aomine*, Mutsuo Hidaka**, Kazuhiro Matsuo*** and Tosio Kitagawa***

\begin{abstract}
Static characteristics of the circuit with three nonidentical Josephson junctions at the centre of triangular sides have been studied experimentally and theoretically. Here as the Josephson junctions Dayem bridges have been used. The measured maximum currents of the circuit under zero voltage are in fairly good agreement with the envelope of the calculated state diagram expressing the thresholds inside which the fluxoid state is stable.
\end{abstract}

\section{Introduction}

The Josephson effect is a fundamental effect of superconductivity, which occurs between two weakly connected superconductors $[1,2]$. Currents can flow depending on a phase difference of wave functions of electrons in two superconductors; in bulky superconductors the phases of wave functions of a great number of electrons are same. The phase difference is controlled by a magnetic field and current. A device having the Josephson effect is called to be a Josephson junction. If it is used as a switching element, it is expected that information bits are processed with ultrahigh switching speed, very low power consumption and signals free from distortion. Many studies of application of Josephson junctions to computer are presently being given.

There are two ways for the Josephson junction to be used as the switching element. One is that the Josephson junction in circuits is changed between zero and nonzero voltage state by applying and reducing a magnetic field or current; the Josephson junction acts as steerage of current path. Another is in the following: Fluxoids ${ }^{1)}$ are put in and out the closed superconducting loop containing Josephson junctions, i.e. the

* Research Institute of Fundamental Information Science, Kyushu University 33, Fukuoka 812, Japan.

** Department of Information Systems, Interdisciplinary Graduate School of Engineering Sciences, Kyushu University 39, Fukuoka 816, Japan.

Present address, Microelectronics Research Laboratories, Nippon Electric Co., Ltd., Kawasaki 213, Japan.

*** International Institute for Advanced Study of Social Information Science, Fujitsu Ltd., Numazu 410-03, Japan.

1) The fluxoid is defined as the sum of the ordinary magnetic flux and a term involving the persistent current around a hole passing through the superconductor. 
fluxoid is employed as information bits. If the fluxoids pass the junction, the phase difference across it changes by $2 \pi$ from that of an initial state. Information processing per unit time in the latter way is about $10^{2}$ times as fast as in the former way. Furthermore power consumption in the latter is $10^{-2}$ times as low as in the former. Recently semiconducting devices with the switching time comparable with the Josephson junctions appear. However, the power dissipated by switching for the semiconducting devices is $10^{4} \sim 10^{5}$ times as large as the way using the fluxoids. The power is emitted as heat. Then this small power consumption in the Josephson junctions makes dense packing possible. Also, the Josephson junctions have characteristics that signals can be transmitted without distortion. This characteristics cannot be attained in the semiconducting devices.

In order to realize the computer using the Josephson junctions, there are unsolved problems which have not so far been experienced in the computer with the semiconducting devices. These problems are the choice of materials, in particular, mechanically robust material, reproducibility of the junction characteristics from batch to batch, circuit design, package of junctions and etc.

We have studied to clarify the properties of several networks containing the Josephson junctions and to apply to new information processing. Some results of measurement and analysis have been reported for the networks with two, three, five and nine junctions $[3,4,5]$. In these circuits two currents which work respectively as a gate and control current flow. The circuit containing the junctions $J_{i}(i=1,2,3)$ being located at the centre of each side of a triangle is shown in Fig. 1.

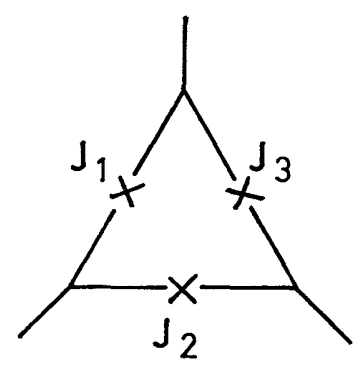

Fig. 1. Circuit with three Josephson junctions.

The experimental current-voltage characteristics of the circuit [5], even if the critical currents of three junctions were almost equal, had voltage appearance of two steps. At the first step two junctions became nonzero from zero voltage state, while at the second step three junctions including the junction, which had remained zero voltage state at the first step, became to have voltage. The first step corresponded to a maximum (critical) current at which the voltage of the circuit was zero; the first step indicated a behaviour of static characteristics. The measured critical current for the first step was in fairly good agreement with that calculated from the equivalent circuit model. Considering from the agreement, the circuit below the critical current for the first step was considered to have fluxoid states.

In this paper, extending the above work about the three identical junctions in the triangular loop to the case of the nonidentical junctions, we report on some further results. 


\section{Experiment}

The Josephson junctions used in this work are constant thickness bridges, i. e. Dayem bridges made from tin. Their thickness and length are 0.2 and $1 \mu \mathrm{m}$, respectively. Their widths of three bridges composing a loop are different from one another and about $1 \mu \mathrm{m}$. The thin films $10 \mu \mathrm{m}$ wide and $0.2 \mu \mathrm{m}$ thick connecting the bridges are shown in Fig. 2. For the fablication of triangular loop containing the bridges, photo-

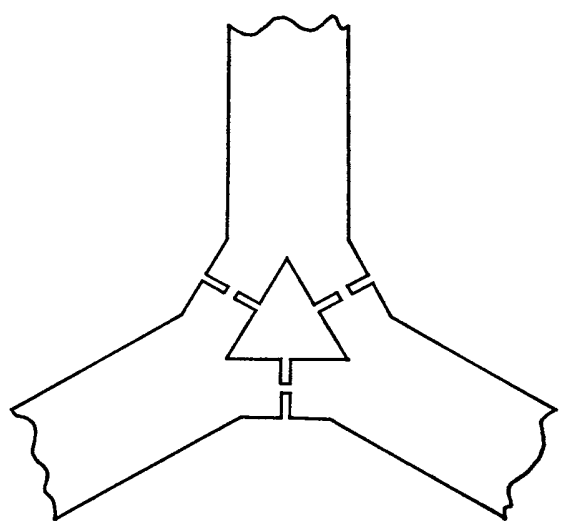

Fig. 2. Schematic representation of circuit pattern in the experiment.

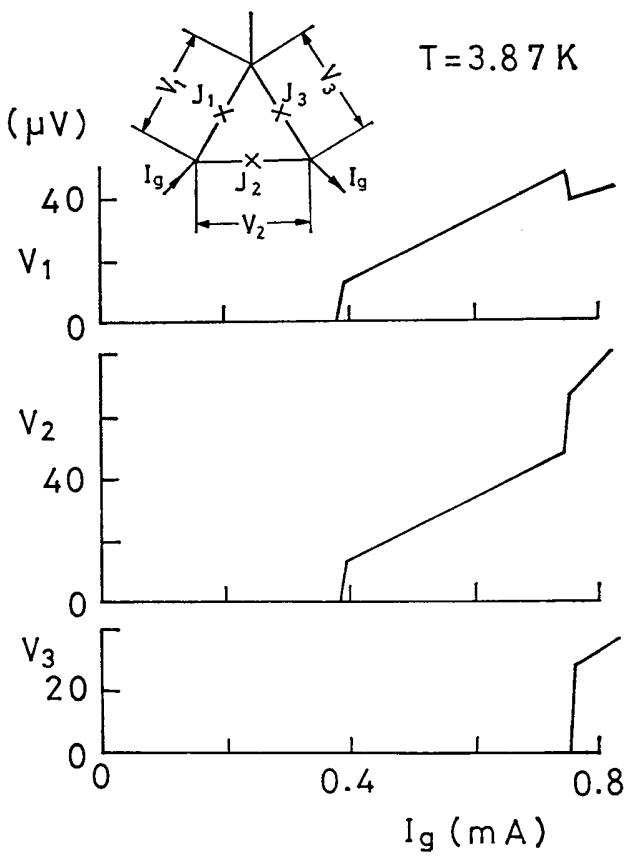

Fig. 3. Current-voltage characteristics of three Dayem bridges. Inset indicates the measurement of the voltages $V_{i}(i=1,2,3)$ across the bridges $J_{i}$ and the passing of the gate current $I_{g}$. The temperature $T$ is $3.87 \mathrm{~K}$. 
lithography, vacuum evaporation and lift-off were used.

Next we describe a method of measurement of current-voltage characteristics. Two d.c. currents were supplied from independent batteries; one is the gate current and another the control current. The voltage was measured using a standard four terminal method and it was plotted against the gate current on an $X-Y$ recorder. A typical example of the measured current-voltage characteristics of the three junctions is shown in Fig. 3. Here the gate current was supplied as in inset and the control current was zero. It is seen from the figure that there exist clearly the first and the second step.

The specimen was immersed in liquid helium. A dewar was surrounded by threefold $\mu$-metal shield which reduced a stray magnetic field. The temperature of specimen was controlled by keeping helium vapour pressure constant and was determined using a carbon resistance thermometer.

\section{Calculation of State Diagram}

A configuration of a set of regions, where the fluxoid states are stable for the gate and control current, is expressed by a state diagram. An equivalent circuit used is shown in Fig. 4.

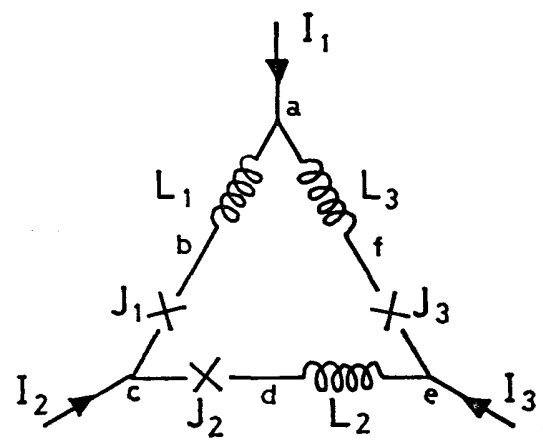

Fig. 4. Equivalent circuit.

Each side of the triangle has a Josephson junction $J_{i}(i=1,2,3)$ with critical current $I_{0 i}(i=1,2,3)$ and inductance $L_{i}(i=1,2,3)$. The currents entering the circuit are denoted by $I_{i}(i=1,2,3)$. The phases of the wave functions at each point in Fig. 4, a, b, c, d, e, $f$ are taken as $\varphi_{M}, \varphi_{1}, 0, \varphi_{2}, \varphi_{N}, \varphi_{3}+\varphi_{N}$, respectively. The Gibbs free energy $G$ for the equivalent circuit of Fig. 4 becomes [6]

$$
\begin{aligned}
G= & \frac{\phi_{0}}{2 \pi}\left[I_{01}\left(1-\cos \varphi_{1}\right)+I_{02}\left(1-\cos \varphi_{2}\right)+I_{03}\left(1-\cos \varphi_{3}\right)+I_{1} \varphi_{M}+I_{3} \varphi_{N}\right. \\
& \left.+\left(\frac{\phi_{0}}{4 \pi L_{1}}\right)\left(\varphi_{1}-\varphi_{M}\right)^{2}+\left(\frac{\phi_{0}}{4 \pi L_{2}}\right)\left(\varphi_{2}-\varphi_{N}\right)^{2}+\left(\frac{\phi_{0}}{2 \pi L_{3}}\right)\left(\varphi_{M}-\varphi_{3}-\varphi_{N}-2 \pi n\right)^{2}\right],
\end{aligned}
$$

where $\phi_{0}$ is a flux quantum and $n$ is a fluxoid number. A maximum current of the circuit under the zero voltage corresponds to $I_{i}$ at which the free energy becomes minimum. The condition for an extremum is

$$
\frac{\partial G}{\partial \varphi_{i}}=0 \text {, }
$$


where

$$
\varphi_{i}=\varphi_{1}, \varphi_{2}, \varphi_{3}, \varphi_{M}, \varphi_{N}
$$

Eliminating $\varphi_{M}, \varphi_{N}$ in the equations obtained from (3.2),

$$
\begin{aligned}
& I_{1}=I_{01} \sin \varphi_{1}-I_{03} \sin \varphi_{3}, \\
& I_{2}=-I_{01} \sin \varphi_{1}+I_{02} \sin \varphi_{2}, \\
& I_{3}=-I_{02} \sin \varphi_{2}+I_{03} \sin \varphi_{3}, \\
& \varphi_{1}+\varphi_{2}+\varphi_{3}+\alpha_{1} \sin \varphi_{1}+\alpha_{2} \sin \varphi_{2}+\alpha_{3} \sin \varphi_{3}=2 \pi n,
\end{aligned}
$$

where

$$
\alpha_{i}=\frac{2 \pi L_{i} I_{0 i}}{\phi_{0}} \quad(i=1,2,3) .
$$

The condition necessary for the solution to be minimum is the following;

$$
\begin{aligned}
g= & p_{1} \cos \varphi_{1} p_{2} \cos \varphi_{2}+p_{2} \cos \varphi_{2} p_{3} \cos \varphi_{3}+p_{3} \cos \varphi_{3} p_{1} \cos \varphi_{1} \\
& +\left(\alpha_{1}+\alpha_{2}+\alpha_{3}\right) p_{1} \cos \varphi_{1} p_{2} \cos \varphi_{2} p_{3} \cos \varphi_{3} \geqq 0,
\end{aligned}
$$

where

$$
p_{i}=\frac{I_{0 i}}{I_{0}} \quad(i=1,2,3)
$$

and

$$
I_{0}=\frac{1}{3} \sum_{i=1}^{3} I_{0 i}
$$

We solve (3.3) under (3.4). The solutions obtained by numerical calculation are expressed by means of the maximum of $I_{i}(i=1,2,3)$ for the control current. We indicate two examples of the calculation in Figs. 5 and 6 , where $I_{3}$ is taken to be $-\left(I_{1}+I_{2}\right)$ and the states for the fluxoid numbers $n$ ranging from 0 to \pm 5 are indicated.

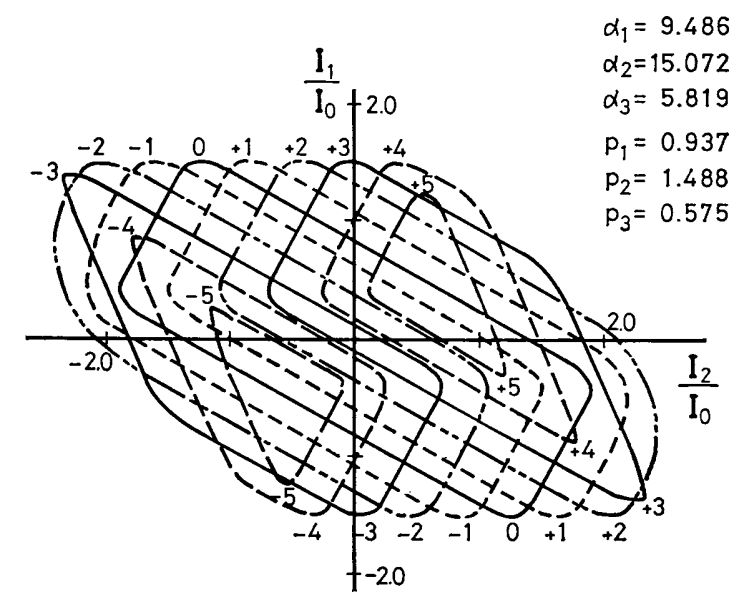

Fig. 5. State diagram for currents $I_{1}$ and $I_{2}$ with three nonidentical junctions. The values of $\alpha_{i}$ and $p_{i}$ are shown in the figure. The numbers indicated near the envelope are the fluxoid number. 


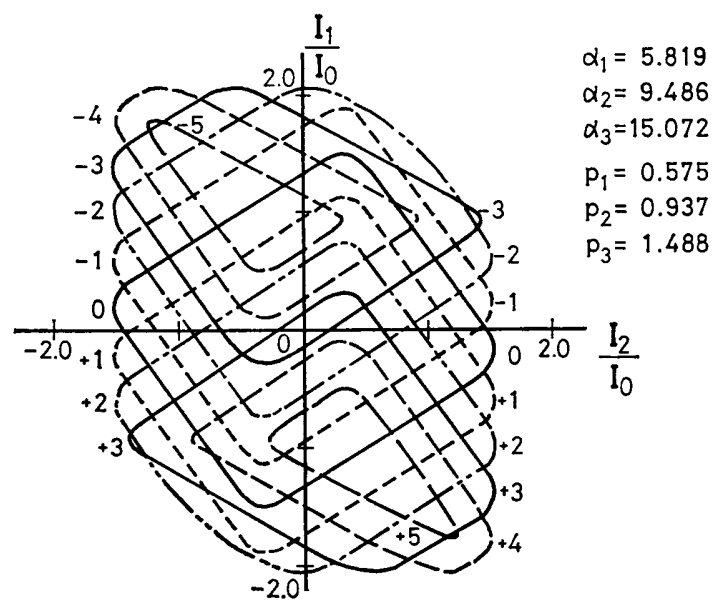

Fig. 6. State diagram for currents $I_{1}$ and $I_{2}$ with three nonidentical junctions. The values of $\alpha_{i}$ and $p_{i}$ are different from those in Fig. 5. The numbers indicated near the envelope are the fluxoid number.

In the figures the lines express the threshold for each fluxoid state; the region surrounded by the lines has a stable fluxoid state corresponding to each $n$.

\section{Comparison of Experiment and Calculation}

It has been found from our work $[4,5]$ that the critical current corresponding to the first step shows an envelope of state diagram. However, in the former work, all the junctions in the circuit were identical. In order to see whether this is also attained in this work about the nonidentical junctions, let us try to compare the experimental critical currents for the first step with envelopes of the calculated state diagram. The results are shown in Figs. 7 and 8, where the experiment and calculation are expressed by open circles and solid lines, respectively.

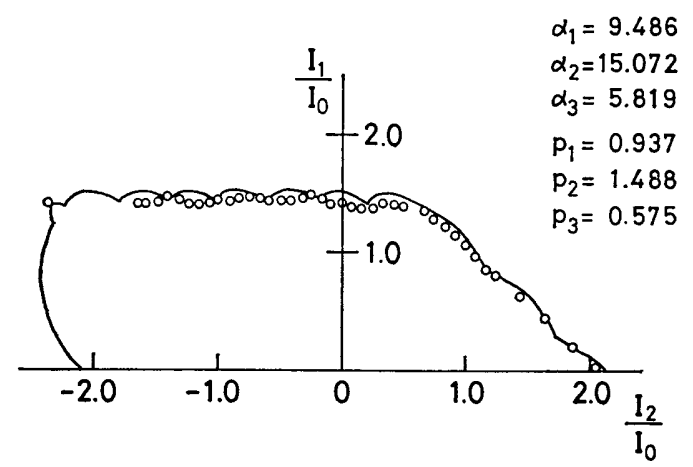

Fig. 7 Comparison of experimental values (open circles) of the critical current for the first step with the calculated envelope (solid line) of the state diagram. The values of $\alpha_{i}$ and $p_{i}$ are same as in Fig. 5. 


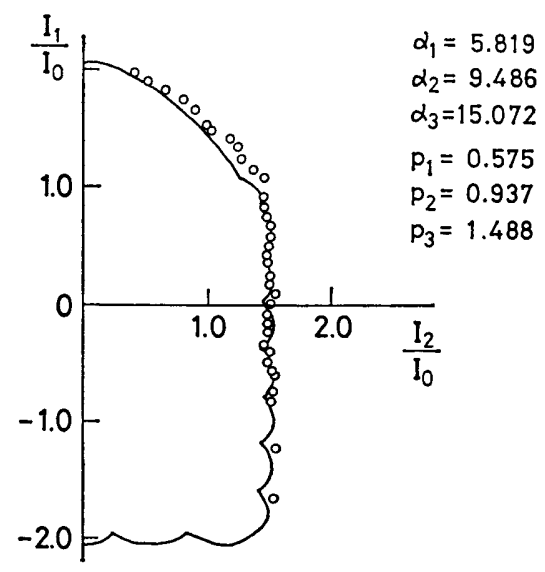

Fig. 8. Comparison of experimental values (open circles) of the critical current for the first step with the calculated envelope (solid line) of the state diagram. The values of $\alpha_{i}$ and $p_{i}$ are same as in Fig. 6.

As seen from the figures, there is fairly good agreement between the experiment and calculation. This fact means, although we used Dayem bridges as Josephson junctions, that the current-phase relation in these bridges follows in such a way as in (3.2) and that the equivalent circuit is reasonable.

\section{Difference in the State Diagrams between Three Identical and Nonidentical Josephson Junctions in the Triangular Loop}

According to the calculated state diagram of the circuit with the three identical junctions [5], the region of state with the fluxoid number $n=0$ has a shape near a hexagon and with increase of $n$ the shape of the region of state becomes a triangle and its centres shrink toward the origin of coordinates. On the other hand in the calculated state diagram of the circuit with the three nonidentical junctions (see Figs. 5 the 6), the shape of the region of state with $n=0$ is almost a tetragon and as $n$ increases, it becomes triangular. In this case the centres of regions with positive sign of $n$ separate from those with negative sign of $n$.

In our experiment a whole structure of the state diagram was not detected but only its envelope was observed. However, considering from the above agreement between the experiment and calculation, even if the thresholds of all the states with each number of fluxoids are measured, the calculated state diagram is expected to be realized.

\section{Acknowledgements}

This work was partially supported by a Grant-in-Aid for Special Project Research from the Ministry of Education, Science and Culture, Japan. 


\section{References}

[1] Josepryson, B. D.: Possible New Effects in Superconductive Tunnelling, Phys. Lett. 1, (1962), 251-253.

[2] Josephison, B. D.: Supercurrents through Barriers, Adv. Phys. 14, (1965), 419-451.

[3] Aomine, T., Mizuno, K., Miyake, K., Matsuo, K., Kusunoki, S. and Hidaka, M. : Effects of Magnetic Field and Injected Current on dc SQUIDs with Dayem Bridges, Jpn. J. Appl. Phys. 20, (1981), 1311-1317.

[4] Aomine, T., Hidaka, M., Kusunoki, S., Miyake, K., Matsuo, K., Kitagawa, T. and Suzuki, C.: Characteristics of Josephson Networks-State Diagram Calculations and Experiments of a Circuit in a Double Loop Structure with Five Dayem Microbridges-, Jpn. J. Appl. Phys. 21, (1982), 490-496.

[5] Aomine, T., Hidaka, M., Matsuo, K. and Kitagawa, T.: Properties of Josephson Networks-Current-Voltage Characteristics of Circuits in Triangular Loops-, Proc. of Sym. on Superconductive Quantum Electronics, (1983), 92-93.

[6] Schultz-DuBois, E. O. and Wolf, P.: Static Characteristics of Josephson Interferometers, Appl. Phys. 16, (1978), 317-338.

Received October 6, 1983 